\title{
Neural Network Based Detection of Melanoma Skin Cancer
}

\author{
Saudamini S. Jivtode ${ }^{1}$, Amit Ukalkar ${ }^{2}$ \\ ${ }^{1}$ Department of Computer Science \\ Kavikulguru Institute of Technology and Science \\ Ramtek, Dist. Nagpur, India \\ ${ }^{2}$ Department of Computer Science \\ Kavikulguru Institute of Technology and Science \\ Ramtek, Dist. Nagpur, India
}

\begin{abstract}
Skin cancer is the deadliest form of cancers in humans. It is found in various types such as Melanoma, Basal and Squamous cell Carcinoma among which Melanoma is the most unpredictable. Detection of melanoma skin cancer in the earlier stage is very critical. In this paper, we explain the method for the detection of Melanoma Skin Cancer using Segmentation. The input to the system is the Dermoscopic Image and then apply and then by applying novel image processing techniques. The different stages of detection involves- collection of Dermoscopic Images, filtering the images by using Dull Razor filtering for removing hairs and air bubbles in the image, converting to gray scale, contrast enhancement, noise filtering, segmenting the images using Maximum Entropy Threshold. Feature Extraction technique used is Gray Level Co-occurrence Matrix (GLCM).It is a powerful tool for image feature extraction by mapping the gray level co-occurrence probabilities based on spatial relations of pixels in different angular directions. They are mean, standard deviation, Skewness, Kurtosis, Contrast, Energy and Homogeneity. Mean or expected value provides a measure of distribution. The sensitivity and specificity for diagnosis of melanoma achieved by neural network analysis of Raman spectra were $85 \%$ and $99 \%$, respectively. We propose that neural network analysis of near-infrared Fourier transform Raman spectra could provide a novel method for rapid, automated skin cancer diagnosis on unstained skin samples.
\end{abstract}

Keywords: Skin cancer, Segmentation, Thresholding, Neural Network, GLCM, Melanoma Detection

\section{Introduction}

Skin is the outermost covering of human body. It is a protective layer of the body which acts as first line of defense against foreign particles entering into the body. There are many diseases or conditions that affect the skin, one such abnormality occurring in skin is skin cancer. Normal cells grow in a controlled way such that new cells replace the old ones. But in the case of cancer, they grow in an abnormal way.

Normal cells become cancerous due to the genetic disorders occurring in the nucleus of the cells by external or internal factors Skin cancer at its early stages can be cured. But when it is not recognized at its early stages, it begins to spread to other parts of the body and can be deadly.

Skin cancer is the most common of all cancer types. In skin cancer number of cases has been going up over the past few decades. Many skin cancers are caused by much exposure to ultraviolet (UV) rays. Most of this exposure comes from the sun and man-made sources.

\section{Stages of Skin Cancer}

The term 'stage of cancer' means the stage the cancer was at when it was first diagnosed. Being sure about the stage is very important because it is a critical factor in deciding the best way to treat the cancer. Stages of skin Cancer are given below [8][9]:

\section{Stage 0}

Stage 0 is also called Bowen's disease or carcinoma. It means the cells are still in the place where they started to develop. So the cells have started to turn into cancer, but they have not yet spread or grown into surrounding areas of the skin. If it is not treated, Bowen's disease can develop into a squamous cell skin cancer. So doctor may describe this stage as pre- cancerous or premalignant.

\section{Stage 1}

Stage 1 means the cancer is 1 to $2 \mathrm{~mm}$ across or less and has 1 or no high risk features. High risk features mean the cancer.

- Is more than $2 \mathrm{~mm}$ thick

- Has grown into the lower dermis

- Has grown into the space around a nerve (peri neural invasion)

- $\quad$ Started on the ear or lip

- Looks very abnormal under the microscope (the cells are poorly differentiated or undifferentiated)

\section{Stage 2}

Stage 2 means the cancer is more than $2 \mathrm{~mm}$ across, or has 2 or more high risk features.

\section{Stage 3}

Stage 3 means the cancer 


\section{International Journal of Science and Research (IJSR) \\ ISSN (Online): 2319-7064}

Index Copernicus Value (2013): 6.14 | Impact Factor (2015): 6.391

- Has grown into the bones in the face, such as the jaw bone or the bone around the eye, OR

- Has spread to a nearby lymph node (or lymph gland) on the same side of the body (and is more than $3 \mathrm{~cm}$ ).

\section{Problem Statement}

Human Cancer is a complex disease caused primarily by genetic instability and accumulation of multiple molecular alternations [1],[2]. Current diagnostic and prognostic classifications do not reflect the whole clinical heterogeneity of tumors and are insufficient to make prediction for successful treatment and patient outcome [3],[4]. Most of the currently applied anti-cancer agents do not greatly differentiate between cancerous and normal cells. In addition cancer is often diagnosed and treated too late, when the cancer cells have already invaded and metastasized into other parts of the body. At the time of clinical presentation, a great percentage of patients with breast, lung, colon, prostate, and ovarian cancer have hidden and over metastatic colonies [5].At this stage, therapeutic modalities are limited in their effectiveness. Due to these problems, cancer has overtaken heart disease as the leading cause death for any age in all over the world.Among many types of cancer, Skin cancers are the most common form of cancers in human [6]. It is severe among the faired-skinned population in Europe, North America, and Australia. There are two major types of skin cancer, name malignant melanoma and non-melanoma (basal cell, squamous cell, and Markel cell carcinomas, etc.) [7].Melanoma is more dangerous and can be fatal if not treated. If melanoma is detected in its early stages, it is highly curable,yet advanced melanoma is lethal. It is wellknown that early finding and treatment of skin cancer can reduce the mortality and morbidity of patients.

Digital Dermoscopy is widely considered as one of the most cost effective means to identify and classify skincancer. An automatic dermoscopy image analysis system [8] has usually three stages: (1) Proper Segmentation, (2) feature extraction and selection, and (3) lesion recognition. The proper segmentation is the most important, since it affects the precision of the subsequent steps. Supervised segmentation is somewhat easy to implement by varying its parameters for variety of lesion shapes, sizes, and colors along with diverse skin types and textures. But the unsupervised segmentation is a difficult task due to the above mentioned properties.

\section{Literature Survey}

In medical field, Melanoma detection is done using clinical screening by a trained dermatologist using the device called dermatoscope and it is done by analyzing the $\mathrm{ABCDE}$ [3] rule. If any trace of melanoma is found in the initial screening, then the patient is referred for further biopsy tests which are done by a trained pathologist. The conventional methods are time consuming, expensive and have many side effects due to biopsy tests. Lack of trained dermatologists is a major issue in conventional melanoma screening methods. Existing automated melanoma detection systems can be used for dermoscopic images. Segmentation is the important step in automated melanoma detection systems to get an accurate result by analyzing only the segmented lesion area.

Existing Melanoma detection systems make use of Statistical Region Merging (SRM) [5], Iterative Stochastic Region Merging [6], Multilevel Thresholding [7] and Color Enhancement and Iterative Segmentation [8]. SRM can be used only for dermoscopic images. It does not consider any texture information and it is noise sensitive. Iterative Stochastic Region Merging method is suitable only for macroscopic images which are robust to noise and artifacts, structural irregularities, illumination variations but the procedure is more complex.

This method of segmentation does not consider texture analysis so this method is less accurate. Multilevel Thresholding is suitable for both dermoscopic and digital images but does not include textural information during segmentation and it is computationally more complex. In Color Enhancement and Iterative Segmentation, texture based segmentation is not used. So it is less accurate and complexity is high due to iterative segmentation.

Existing Melanoma detection systems are less accurate and do not include texture analysis for segmentation of skin lesion from the background skin. Texture based segmentation enables accurate segmentation of skin lesion and feature extraction. Classification of the segmented images must be done to accurately classify the images as melanoma or not after extracting various features of skin lesion. Textural based segmentation gives accurate classification results and improves accuracy of the overall melanoma detection system.

\section{Proposed System}

Computer aided melanoma detection system using texture based segmentation and classification is proposed in this work. The proposed framework is shown in Figure1.

This system consists of different modules which include preprocessing, segmentation, feature extraction and classification. Preprocessing is done using histogram equalization.

The preprocessed image is segmented using Texture Distinctiveness Lesion Segmentation [2] (TDLS) method to extract the lesion area from the background skin. The extracted lesion area is used for feature extraction which is done by gray level co-occurrence matrix (GLCM). Using the extracted features, the system is trained using ANN classifier in order to classify the images as malignant or benign melanoma. The melanoma images are again categorized as Superficial Spreading Melanoma, [4] Nodular Melanoma, Lentigo Maligna Melanoma. 


\section{International Journal of Science and Research (IJSR) \\ ISSN (Online): 2319-7064}

Index Copernicus Value (2013): 6.14 | Impact Factor (2015): 6.391

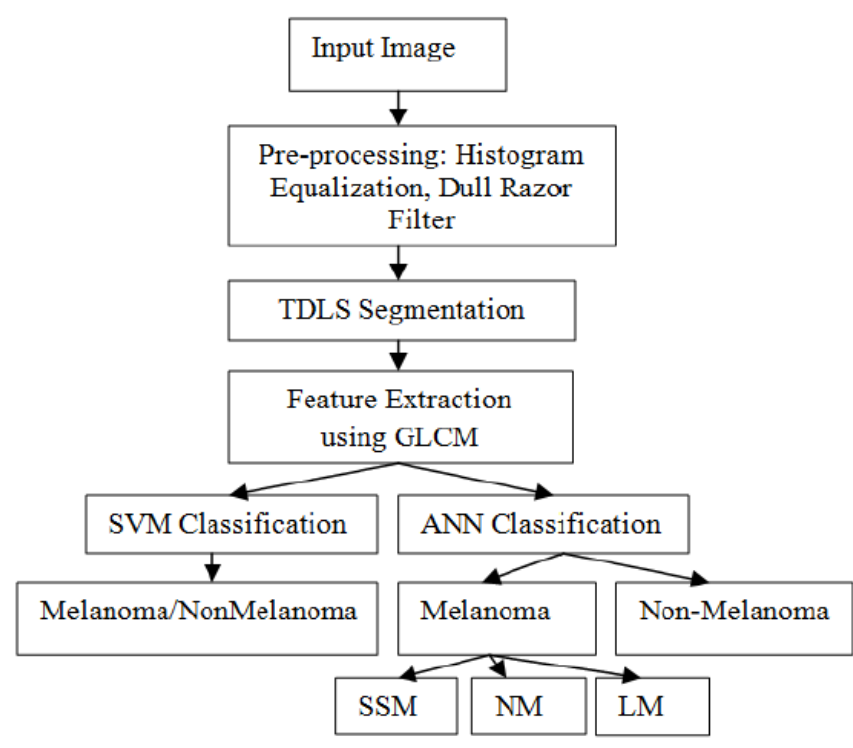

Figure 1: Proposed System

\section{A. Image Acquisition}

Image acquisition is defined as the process of capturing or retrieving an image from a camera, so it can be passed through various processes need to occur later. Image acquisition in image processing is the first step in the workflow sequence since, without an image, no further process is possible.

\section{B. Image Pre-processing}

The pre-processing is a modification of the image data that removes unwanted distortions or enhances some image features important for further processing. First, the image is resized to adjust images to a uniform scale $(512 \times 512)$ so that it supports feature classification with accuracy. After that, images are converted from RGB (Red, Green, and Blue) to grey level where the features are based on grey level co-occurrence matrix (GLCM).

\section{Image Segmentation}

Segmentation of image deals with the process of partitioning a digital image into multiple segments (sets of different pixels) the goal of this process is to simplify and/or change the representation of an image into something that is more meaningful and easier to analyze.[16]. Image segmentation is typically used to locate objects and boundaries (lines, curves, etc.) in images.

\section{Feature Extraction}

Feature extraction is to reduce the original data set by measuring certain features, or properties, that distinguish between input patterns. The features extracted in this work are based on texture analysis using GLCM. The GLCM is a powerful tool for image feature extraction by mapping the grey level co-occurrence probabilities based on spatial relations of pixels in different angular directions. The features extracted from GLCM are: Energy, Correlation Homogeneity, Contrast, Along with other statistical parameters such as skewness, kurtosis and mean.

\section{E. Classification Using Artificial Neural Network}

The segmented image is classified based on the extracted features, such as, the color and texture. The design of classifiers were investigated in many studies, most of them are Artificial Neural Networks (ANN). The neural network classifier is used for classifying Malignant Melanoma from other skin diseases such as benign. Based on the computational simplicity ANN based classifier is used [17]. ANN is able to solve highly complex problems due to the nonlinear processing capabilities of its nodes (neurons). In this proposed system, a feed forward multilayer network is used and Back propagation (BPN) Algorithm is used for training. The neural network structure consists of Input layer, in the middle Hidden layer and Output layer. The hidden layer and output layer adjusts weights value based on the error output in classification of different features. In Back Propagation algorithm, signal flow will be in forward direction. Each time output of the network is compared with desired output, if both do not match; an error signal is generated at the output.

This error signal is propagated backwards and weights are adjusted at the middle layer so as to reduce the error. In this Back propagation algorithm, the hidden layer and output layer weights are initialized randomly at the beginning of training. Supervisory learning process is used for training. During forward pass of the input signal, according to the initial weights and activation function used, the neural network gives an output and is compared with required output. If both do not match, an error occurs. During reverse pass, the error signal is back-propagated and weights of both hidden and output layer are adjusted. The whole process of training will continues until error is zero. The neural network is trained with desired known values.

After training, neural network can perform decision making. Matlab is used for implementation including image preprocessing and for artificial neural network classification.

The neural network is trained using the known statistical values and dermoscopic feature of melanoma images. The statistical values and dermoscopy feature of around 60 melanoma cancerous images and non-cancerous images are calculated and given to the classifier, which will give output as either 0 or 1 .

\section{Modules}

\section{A. Dermoscopy}

Dermoscopy is also known as Dermatoscopy or Epiluminescence Light Microscopy (ELM). The image obtained from such a dermatoscope is called Dermoscopic Image.

\section{B. Dull Razor Filtering}

The Dermoscopic Images are in Digital format. Preprocessing is done to removes the noise, fine hair and

\section{Volume 5 Issue 6, June 2016 www.ijsr.net}




\section{International Journal of Science and Research (IJSR) \\ ISSN (Online): 2319-7064}

Index Copernicus Value (2013): 6.14 | Impact Factor (2015): 6.391

bubbles in the image. The Hair removal is done here by using Dull Razor Filter.

\section{Converting to gray scale}

The standard image size is taken as $360 \times 360$ pixels. Before preprocessing, the color cancer image converted into grayscale image by eliminating hue and saturation. The algorithm is to convert RGB values to grayscale values by forming a weighted sum of $R, G$ and $B$ Component.

\section{Contrast Enhancement}

In this step, we are trying to increase image clarity and obtain better performance. It is done to enhance the shape and edges of image. In addition, contrast enhancement can sharpen the image border and improve the accuracy for segmentation.

\section{E. Noise Filtering}

We used Median Filtering to remove noise. Median filtering is used for minimizing the influence of small structures like thin hairs and isolated islands of pixels like small air bubbles. It is used to remove pepper and slat noise.

\section{F. Feature Extraction}

Feature Extraction technique used is gray Level Cooccurrence Matrix (GLCM).It is a powerful tool for image feature extraction by mapping the gray level co-occurrence probabilities based on spatial relations of pixels in different angular directions. They are mean, standard deviation, skewness, Kurtosis, Contrast, Energy, Homogeneity. Mean or expected value provides a measure of distribution

\section{G. Classifier}

Classifier is used for classifying skin cancer.

\section{Conclusion}

Melanoma detection in the initial stage is very important since it can reduce the death rates to a great extent. Early detection can be done only with the help of trained dermatologists and proper diagnosis. Due to the lack of trained dermatologists, expensive and time consuming diagnosis procedures, automated system for melanoma detection is required. An automated melanoma detection system using texture based lesion segmentation, feature extraction using GLCM and classification of images using SVM and ANN classifiers is implemented. Using ANN classifier, melanoma images are again classified into 3 categories namely Superficial Spreading Melanoma, Nodular Melanoma and Lentigo Maligna Melanoma. This proposed system can gives better diagnosis and accuracy than conventional clinical screening and biopsy tests since it makes use of texture based analysis and classification.

\section{REFERENCES}

[1] Details of skin cancer. [Online]. Available at: http://www.cancer.gov/cancertopics/types/skin

[2] J. Glaister, A. Wong, and D. A. Clausi, "Segmentation of Skin Lesions From Digital Images Using Joint Statistical Texture Distinctiveness," IEEE Trans. Biomed. Eng., vol. 61, no. 4,pp. 1220-1231, Apr. 2014

[3] Melanoma Available

at: http://www.skincancer.org/skin-cancerinformation/melanoma

[4] Types of Melanoma. [Online]. Available at: http://www.ncbi.nlm.nih.gov/pmc/articles/PMC32539 44.

[5] M. E. Celebi, H. A. Kingravi, H. Iyatomi, Y. A. Aslandogan, W. V. Stoecker, R. H. Moss, J. M. Malters, J. M. Grichnik, A. A. Marghoob, H. S. Rabinovitz, and S. W. Menzies, "Border detection in dermoscopy images using statistical region merging," Skin Res. Technol., vol. 14, no. 3, pp. 347-353, 2008.

[6] J. Glaister, A. Wong, and D. A. Clausi , "Automatic Skin Lesion Segmentation via Iterative Stochastic Region Merging," IEEE trans. Biomed. Eng.,vol15,No.6,Nov 2011

[7] J.Humayun , A.S.Malik , N.Kamel , "Multilevel thresholding for segmentation of pigmented skin lesions, " IEEE Int. Conf. Imaging Systems and Techniques, pp. 310-314,May. 2011

[8] G.Schaefer, M. Rajab , M. Emre Celebi and Hitoshi Iyatomi, "Skin lesion extraction in dermoscopic images based on colour enhancement and iterative segmentation," ICIP ,IEEE Journal,pp.33613364,2009

[9] Dull Razor Filter. [online]. Available at: http://www.dermweb.com/dull_razor/

[10] N.Hema Rajini , R.Bhavani, "Computer aided detetcion of ischemic stroke using segmentation and texture features" ELSEVIER, Measurement 46, pp.1865-1874,2013

[11]Guoqiang Peter Zhang, "Neural Networks for Classification", IEEE Transactions On Systems, Man, And Cybernetics-Part C: Applications And Reviews, Vol. 30,2000

[12]Feed Forward Network. Available at: http://www.fon.hum.uva.nl/praat/manual/Feedforward _neural_networks_1__What_is_a_feedforward_ne.ht $\mathrm{ml}$

[13] DermQuest Image Database [online]. Available at: https://www.dermquest.com/results/?q=Malignant $\% 2$ Omelanoma

[14]P. G. Cavalcanti, J. Scharcanski, andC. B. O. Lopes, "Shading attenuation in human skin color images," in Advances in Visual Computing, G. Bebis, R. Boyle, B. Parvin, D. Koracin, R. Chung, R. Hammoud, M. Hussain, T. Kar-Han, R. Crawfis, D. Thalmann, D. Kao, and L. Avila, Eds., (ser.Lecture Notes in Computer Science), vol. 6453 Heidelberg, Germany: Springer, 2010, pp. 190-198.

[15]P. G. Cavalcanti and J. Scharcanski, "Automated prescreening of pigmented skin lesions using standard cameras," Comput.Med. Imag. Graph., vol. 35, no. 6, pp. 481-491, Sep. 2011.

\section{Volume 5 Issue 6, June 2016 www.ijsr.net}




\section{International Journal of Science and Research (IJSR) \\ ISSN (Online): 2319-7064}

Index Copernicus Value (2013): 6.14 | Impact Factor (2015): 6.391

[16] Maurya R, Surya K . S , Maurya K .A and Ajeet ," GLCM and Multi Class Support Vector Machine based Automated Skin Cancer Classification,"IEEE journal,vol 12,pp 444-447, 2014.

[17] Messadi M, Cherifi H and Bessaid A," Segmentation and ABCD rule extraction for skin tumors classification," Journal of Convergence Information Technology (JCIT), Volume9, Number2, March 2014.

[18]Parekh R ,"Using Texture Analysis for Medical Diagnosis," IEEE Computer Society, pp 28-37, apriljune 2012.

[19] Raja J.V.C, Jeyaprakash M," Skin Disease Diagnosis Using Texture Analysis," International Journal of Advanced Research in Computer Science and Software Engineering, IJARCSSE, Volume 4, Issue 1, pp 275-278, January 2014.

[20] Rajam P.J.J, Thasneem H.A.A," Detection of Skin Lesions in Dermoscopic Images," International Journal of Recent Development in Engineering and Technology, Volume 2, Special Issue 3,pp 193-198, February 2014.

[21] Ramteke S.N and Shweta V.J ," Analysis of Skin Cancer Using Fuzzy and WaveletTechnique - Review \& Proposed New Algorithm," International Journal of Engineering Trends and Technology (IJETT), Volume 4 , Issue 6 , june 20

Volume 5 Issue 6, June 2016 www.ijsr.net 